\title{
Low Frequency Radio Astronomy from Above the Ionosphere
}

\author{
D. L. Jones
}

Jet Propulsion Laboratory, California Institute of Technology, Mail Code 238-332, 4800 Oak Grove Drive, Pasadena, CA 91109, USA

for the ALFA Midex team: R. Allen (STScI), J. Basart (Iowa State), T. Bastian (NRAO), W. Blume (JPL), J.-L. Bougeret (Obs. Paris), B. Dennison (VPI), M. Desch (GSFC), K. Dwarakanath (RRI), W. Erickson (U. Md. \& U. Tasmania), W. Farrell (GSFC), D. Finley (NRAO), N. Gopalswamy (GSFC), R. Howard (Orbital), M. Kaiser (GSFC), N. Kassim (NRL), T. Kuiper (JPL), R. MacDowall (GSFC), M. Mahoney (JPL), R. Perley (NRAO), R. Preston (JPL), M. Reiner (GSFC), P. Rodriguez (NRL), R. Stone (GSFC), S. Unwin (JPL), K. Weiler (NRL), G. Woan (U. Glasgow), and R. Woo (JPL)

\begin{abstract}
The GMRT represents a dramatic improvement in groundbased observing capabilities for low frequency radio astronomy. At sufficiently low frequencies, however, no ground-based facility will be able to produce high resolution images while looking through the ionosphere. A space-based array will be needed to explore the objects and processes which dominate the sky at the lowest radio frequencies. An imaging radio interferometer based on a large number of small, inexpensive satellites would be able to track solar radio bursts associated with coronal mass ejections out to the distance of Earth, determine the frequency and duration of early epochs of nonthermal activity in galaxies, and provide unique information about the interstellar medium.
\end{abstract}

\section{Introduction}

Ground-based radio interferometers, now augmented by the GMRT, are able to produce images of the sky at frequencies down to a few tens of MHz. Some important scientific goals, however, require imaging at even lower frequencies. Absorption and refraction by the ionosphere prevents imaging from the ground at frequencies of a few $\mathrm{MHz}$ and lower, so an interferometer array composed of inexpensive satellites will be needed. Suitable locations for a space-based array include very high Earth orbits, halo orbits about the Sun-Earth Lagrange points, Earth-trailing heliocentric orbits, and the far side of the moon. The optimal choice depends on financial considerations and the unavoidable tradeoff between a benign environment in which to maintain a multi-satellite array and the difficulty of getting enough data from the array to Earth. 


\section{Science Goals}

What unique science can be done only at frequencies below $\sim 10 \mathrm{MHz}$ ? There are two general areas where very low frequency observations are critical: First, sources of emission which are intrinsically limited to low frequencies (e.g., plasma oscillations and electron cyclotron masers), and second, observations of strongly frequency-dependent absorption (e.g., free-free absorption by diffuse ionized interstellar hydrogen). Type II radio bursts from interplanetary shocks driven by coronal mass ejections provide a good example of the first case. These intrinsically narrow-band emissions decrease in frequency as the shock propagates farther from the sun into regions of lower plasma density. In order to image and track type II bursts as they approach $1 \mathrm{AU}$ from the sun, observations at frequencies below $1 \mathrm{MHz}$ are necessary. This would allow us to predict the arrival at Earth of coronal mass ejections, which can trigger severe geomagnetic storms.

A sensitive map of the radio sky with arcminute angular resolution at a few $\mathrm{MHz}$ would be especially effective at detecting coherent emission from disks, jets, and possibly gas giant planets orbiting close to nearby stars. In addition, diffuse ionized hydrogen could be detected via its absorption of radiation from extragalactic radio sources across the sky. These observations would complement $\mathrm{H} \alpha$ emission maps, which predict large variations in free-free optical depth on angular scales of a few degrees. All-sky surveys at low frequencies would map the galactic distribution of low energy cosmic ray electrons and would likely discover large numbers of high redshift galaxies, "fossil" radio lobes, and largescale shocks and shells from old galactic supernovae and $\gamma$-ray bursts.

\section{Requirements for a Space-based Array}

Any space-based array for very low frequency imaging will need to meet three fundamental requirements: 1) the array must be located far enough from Earth to avoid terrestrial interference and the extended ionosphere, 2) there must be a large enough number of individual antennas in the array to produce dense, uniform $(u, v)$ coverage in all directions simultaneously, and 3) the observing bandwidth must be sufficient to provide useful sensitivity for short snapshot observations. The second and third requirements result from the nearly omnidirectional nature of reasonably sized antennas at very low frequencies. Strong variable radio sources anywhere on the sky will affect the observed total power levels, and unless such sources are imaged on short time scales their time-variable sidelobes will limit the dynamic range of observations in other directions.

\section{Conclusions}

There are compelling scientific reasons to place an imaging low frequency radio array in space. The technology to build and operate such an array affordably is available. The major remaining task is to educate the general astronomical community about the importance of high resolution radio images at low frequencies.

Acknowledgments. Part of this work was carried out at the Jet Propulsion Laboratory, California Institute of Technology, under contract with the US National Aeronautics and Space Administration. 